\title{
Design and Deformation Analysis of Six-component Wheel Dynamometer
}

\author{
Changhwan Shin, Hyunchang Yoo, Jinwon Joo \\ Chungbuk National University \\ 1 Chungdae-ro, Seowon-gu, Cheongju, Chungbuk, 362-763 Korea \\ meidno@naver.com; hyunandyoo@hanmail.net; jinwon@chungbuk.ac.kr
}

\section{Extended Abstract}

A wheel dynamometer is used to measure dynamic load that is transmitted from the road surface to vehicles while driving. In this paper, a six-component (three forces and three moments) wheel dynamometer utilizing shear deformation was designed and evaluated. The cross type structure having four or six radial bars was used as a basic sensing structure of the wheel dynamometer. The body of the dynamometer consists of a rigid central hub, a rigid outer annular ring and the radial bars extending between the hub and the ring. Although most of sensors are mounted conventionally to provide an output signal indicative of bending strains in the sensing bar, other forms of sensors such as those that provide an indication of shear strains was used in this paper for obtaining more stable signal against inevitable location error at bonding strain gauges.

One aspect of the load transducer includes a structure of placing flexural members which are compliant for displacement of each corresponding radial bars. The flexural members on the end of sensing bars in the annular ring serve to produce sufficient shear strain of the sensing bars which lie at 90 degrees, but they can provide non-uniform distributions of shear strain. Since there is a possibility that such a non-uniform strain distribution can cause mutual coupling errors between the forces or moments, deformation analysis is needed thereof. Finite element analysis was performed to design the shape including flexural members and determine the size of sensing parts, and to predict output signals. In order to obtain adequate output signal and minimize coupling errors, the locations of strain gauges and their connection were determined so that Wheatstone bridge circuits with four or eight strain gauges would be balanced. Through the design modifications of the various shapes of flexural members, the coupling effect was minimized. The maximum capacity of the six-component wheel dynamometer designed in this paper is $25 \mathrm{kN}$ in each force Fx, Fy, $20 \mathrm{kN}$ in $\mathrm{Fz}$ and $5 \mathrm{kN} \cdot \mathrm{m}$ in each moment. Strain analysis was performed repeatedly to obtain about $250 \sim 300 \mu \mathrm{m} / \mathrm{m}$ of similar output strain for each maximum load component.

Another aspect of the present transducer include a method of constructing Wheatstone bridge circuit and of signal processing for determining each applied force/moment. Output signals from the strain sensing bars are indicative of force and moment components transmitted between the central hub and the annular ring in six degrees of freedom. Traditionally, six full bridge circuits which are composed of 4 or 8 different selected strain gages are used for the measurement of sixcomponent force/moment. In this case, however, considerable coupling errors have been found when the transducers were actually fabricated and estimated. In this paper, for practical improvement, the transducer measures multiple forces independently on the plurality of sensing bars, and these forces are then transformed to provide forces along and moments about the axes of the coordinate system.

Design modifications resulted in similar strain sensitivities for all six-component forces and moments and reduced coupling errors. A six-component wheel dynamometer made of high-strength aluminium alloy was fabricated with strain gauges for sensing shear strain. Calibration tests were performed to evaluate sensitivities, coupling errors and nonlinearity errors. 